\title{
HIV and SARS-CoV-2: Tracing a Path of Vaccine Research and Development
}

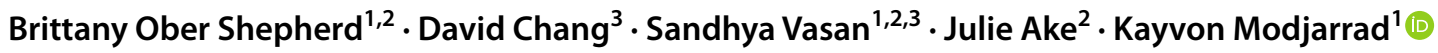

Accepted: 9 December 2021 / Published online: 28 January 2022

This is a U.S. government work and not under copyright protection in the U.S.; foreign copyright protection may apply 2021

\begin{abstract}
Purpose of Review This review examines the major advances and obstacles in the field of HIV vaccine research as they pertain to informing the development of vaccines against SARS-CoV-2.

Recent Findings Although the field of HIV research has yet to deliver a licensed vaccine, the technologies developed and knowledge gained in basic scientific disciplines, translational research, and community engagement have positively impacted the development of vaccines for other viruses, most notably and recently for SARS-CoV-2. These advances include the advent of viral vectors and mRNA as vaccine delivery platforms; the use of structural biology for immunogen design; an emergence of novel adjuvant formulations; a more sophisticated understanding of viral phylogenetics; improvements in the development and harmonization of accurate assays for vaccine immunogenicity; and maturation of the fields of bioethics and community engagement for clinical trials conducted in diverse populations.

Summary Decades of foundational research and investments into HIV biology, though yet to yield an authorized or approved vaccine for HIV/AIDS, have now paid dividends in the rapid development of safe and effective SARS-CoV-2 vaccines. This latter success presents an opportunity for feedback on improved pathways for development of safe and efficacious vaccines against HIV and other pathogens.
\end{abstract}

Keywords SARS-COV-2 $\cdot$ HIV $\cdot$ Vaccine $\cdot$ Research $\cdot$ Lessons

\section{Introduction}

The identification, in December 2019, of a novel human coronavirus, later labeled severe acute respiratory syndrome coronavirus-2 (SARS-CoV-2), represented the beginning of one the greatest public health challenges the world has faced since the emergence of human immunodeficiency virus (HIV) four decades earlier. As the pandemic of coronavirus

This article is part of the Topical Collection on The Global Epidemic

Kayvon Modjarrad

kayvon.modjarrad.civ@mail.mil

1 Emerging Infectious Diseases Branch, Walter Reed Army Institute of Research, 503 Robert Grant Avenue, Suite 2A14, Silver Spring, MD 20910, USA

2 Henry M. Jackson Foundation for the Advancement of Military Medicine, Bethesda, MD 20817, USA

3 US Military HIV Research Program, Walter Reed Army Institute of Research, Silver Spring, MD 20910, USA disease 2019 (COVID-19) has unfolded, the tools and knowledge gained in the 40-year fight against HIV and acquired immunodeficiency syndrome (AIDS) have been critical for the rapid development of reliable countermeasures against COVID-19, including several safe and effective vaccines.

Although a licensed vaccine has eluded the HIV research community, sustained investments into understanding the virus's biology and its interaction with the human host have resulted in a combination of highly effective therapies and prevention modalities that have made significant inroads into the control of the AIDS pandemic. In contrast, the efforts to create an effective vaccine or definitive cure for HIV have been unsuccessful thus far. Nonetheless, global initiatives toward these ends have ushered in a period of creativity and coordination on a scale not seen before in the scientific and public health communities. The unprecedented speed with which SARS-CoV-2 prophylactic and treatment options have been made available to the public was built on this preceding era of innovation in response to HIV/AIDS. In this brief review, we highlight some of the most critical scientific advances and public health lessons gained in the endeavor 
for an HIV vaccine and trace their influence on the accelerated development of multiple COVID-19 vaccines.

\section{Vaccine Platforms}

The history of HIV vaccine development is punctuated by an alternating series of accomplishments and disappointments. Despite the inconsistency of major advances in the field, there has been continuous, steady progress in novel antigen delivery platforms, particularly in the form of genetic vaccines $[1,2]$. DNA, mRNA, and viral vectors-advanced to various stages of clinical evaluation prior to the COVID-19 pandemic - have the advantage of being scalable and customizable, allowing for facile modification to deliver antigens from different pathogens.

Viruses, by definition, can be reduced to self-replicating strings of nucleic acids encased in a protein coat that are dependent on host cell machinery to generate copies of themselves. The evolved properties that define the virus life cycle have been leveraged as an efficient means of delivering genetic sequences of antigens for endogenous production. Multiple virus families have been explored for their ability to serve as shuttles for antigenic sequences. In the context of HIV vaccine research, however, adenoviruses were identified early on as a promising platform to recruit both humoral and cellular arms of the immune response. Two types of adenoviruses, in particular, have been the focus of HIV vaccine platforms: an adenovirus that infects chimpanzees [3, 4] and a specific but rare serotype that infects humans [5]. Both viruses have since been used as viral vectors for two SARS-CoV-2 vaccines that are currently in use worldwide (ChAdOx-1 nCoV-19 and Ad26.COV2.S) [6, 7]. Although intended to be used as standalone monovalent vaccines against the wild-type virus, these platforms can be modified to address variants of concern either in homologous or heterologous prime-boost regimens to recall and potentiate a broader humoral and cell-mediated response against multiple SARS-CoV-2 strains.

Despite their success in reaching the commercial market for SARS-CoV-2, a rare risk of the adenovirus vector platform has since been revealed due to its administration to millions of people. The risk of coagulopathy from the platform, though serious, is so rare that it would never have been identified from the tens of thousands of individuals who had received the vaccine platform in the context of the more recent COVID-19 and prior HIV clinical trials. This newly identified side effect will likely influence the design and conduct of clinical trials with adenovirus vector vaccines for HIV and other pathogens going forward. The utility of adenovirus vaccines for HIV, however, remains unclear. Two efficacy trials, called Imbokodo (HVTN 705) and Mosaico (HVTN 706), are testing an approach that primes with the
Ad26 vector containing mosaic inserts representing multiple HIV subtypes, followed by a protein boost, which also features a mosaic design. The Imbokodo trial demonstrated an efficacy of $25 \%$ with a confidence interval lower bound less than zero. The chimpanzee adenovirus ChAdOx1 is also being evaluated in a heterologous prime-boost strategy, but as a therapeutic vaccine series designed to control HIV-1 viral replication [8].

Perhaps even a greater leap forward in genetic vaccines has been for mRNA platforms, which, despite their more recent widespread recognition, have a history extending back decades [1]. Improvements in mRNA stability, delivery, and expression have been gradual and were made in the context of vaccine development for HIV and other emerging pathogens, such as the Zika virus. The progress, though incremental, was sufficient for the platform's large-scale debut during the COVID-19 pandemic. As such, two highly effective mRNA vaccines have now received either FDA emergency authorization or full approval $[9,10]$.

\section{Structure-Based Approach to Antigen Design}

The platform, though a critical component to any vaccine, is only one part of the product. The antigen that is presented on or delivered through any platform will dictate the specificity of the immune response and largely direct the durability and breadth of immunity. In the early days of the HIV epidemic, vaccine developers took a standard approach in vaccinology, focusing on the wild-type form of the viral surface proteins as the primary immunogens for development. It became clear that the genetic and antigenic variability of HIV, particularly its surface envelope glycoprotein, would preclude this strategy from being a successful one [11]. A more rational approach toward immunogen design eventually took hold, based on the structural resolution of key epitopes that were the target of potent, broadly neutralizing antibodies, which in many viral pathogen models have been a strong correlate of protection.

In recent years, structural biology and reverse vaccinology have become powerful tools to influence antigen potency. These approaches optimize the immunogenicity of antigens by resolving the structural details of surface proteins as a way to engineer key epitopes as targets of the most potent neutralizing antibodies, particularly those identified after natural infection [12-14]. Much of the focus in rational vaccine design for enveloped viruses, like HIV, has been on stabilizing their surface class I fusion proteins in a prefusion conformation. By honing in on this region, vaccine designers have been able to improve the breadth of neutralization [15].

One application of this HIV vaccine design strategy was in scaffolding epitopes to elicit antibody responses against 
the membrane proximal external region of the envelope gp41 subunit [16]. In this approach, a broadly neutralizing epitope was positioned, in its native conformation, onto a heterologous protein scaffold. This translated into the stabilization of the envelope ectodomain in its trimeric prefusion conformation and the generation of the BG505 SOSIP.664 proteins that contain the targets of broadly neutralizing antibodies $[17,18]$. The methods of reverse vaccinology have also been used to understand and improve upon modestly successful vaccine strategies-such as that observed in the seminal RV144 trial and its immune correlates analysis, which revealed antibody responses to the gp120 V1/V2 region correlated inversely with infection risk [19]. While the vaccine candidates used for the prime-boost regimen were not developed by structure-based design, this method is being used by some laboratories for the rational development of subsequent candidates [20-22] as a way to engineer immunogens capable of inducing even more broadly neutralizing antibody repertoires.

Although the methods described above have advanced the science of HIV vaccinology, the greatest leap forward in the use of structural biology for the design of viral surface protein immunogens occurred in the resolution and stabilization of the fusion (F) protein of respiratory syncytial virus (RSV), a major cause of pneumonia in children and the elderly [23-27]. The work with RSV, though owing some of its success to technologies developed through investigations into HIV immunology, has created gains that have reciprocally informed efforts in HIV vaccine research as well as coronavirus vaccine development. Most notably, the evolution of the science culminated in early 2020, with the structural resolution of the SARS-CoV-2 prefusion Spike glycoprotein and its rapid translation into the key immunogen used in the most effective COVID-19 vaccines [28]. The advances made in structural biology and reverse vaccinology for HIV, RSV, and SARS-CoV-2 are now expanding to vaccine designs for other pathogens, like influenza and meningococcus, and are precipitating a paradigm shift in the entire field of vaccine development.

\section{Adjuvants}

In the case of genetic vaccines, the platform and the antigen are the primary components. For many licensed vaccines, particularly recombinant proteins, however, there is another important variable that is key to influencing vaccine-elicited immunity: the adjuvant. Both failures and successes in HIV vaccine development have paved the way for the design and discovery of more potent adjuvants. One of the key lessons learned has been that aluminum hydroxide is a poor adjuvant for HIV-1 antigens in humans [29-33]. Unfortunately, MF59 and saponin-based adjuvants have not provided additional benefit in conferring protective efficacy [34-38]. However, new formulations have been developed in hopes of improving the magnitude and the quality of both the antibody- and cell-mediated immune responses [39]. Furthermore, newer saponin formulations have been shown to induce durable and balanced response profiles [40]. Despite the substandard performance with HIV vaccines, aluminum adjuvants were still tested in combination with SARS-CoV-2 vaccines such as the inactivated virus vaccines, BBIBP-CorV, and CoronaVac, which provided near $100 \%$ protection against severe COVID-19 disease but significantly lower efficacy against infection.

Some of the greatest advances in adjuvant science have come out of investments into liposomal formulations that have been used in concert with antigens across a range of pathogens. Liposomal adjuvants are now being used for two COVID-19 vaccines, including Matrix-M for the Novavax vaccine candidate and ALFQ for the Army's Spike ferritin nanoparticle $(\mathrm{SpFN})$ vaccine candidate, both of which contain monophosphoryl lipid A (MPLA) and the saponin QS-21 [41-44]. ALFQ now has been in three phase I clinical trials and a third that is likely to begin at the end of 2021 with HIV-1 A244 antigen [45]. The science of adjuvant design and development across the field of vaccinology will likely be guided by the outcomes of these and other nextgeneration SARS-CoV-2 recombinant protein vaccines as more data on their efficacy and durability become available.

\section{Prime-Boost Strategies}

The immunization schedule and the potential use of different vaccine platforms in that schedule have the potential to strongly influence immunologic memory to a particular antigen. These so-called prime-boost strategies have been evaluated for several decades through modifications of the type, delivery, and timing of immunogens administered as a means toward enhancing both the strength and diversity of the humoral and cell-mediated immune responses. Heterologous prime-boost regimens, though investigated in the early years of the HIV epidemic, had lost momentum as a viable strategy until 2009 when the positive results of the RV144 trial were reported. To date, this is the only trial that demonstrated statistically significant efficacy in preventing HIV acquisition, with a point estimate of $31 \%$ at 3.5 years [46] and $60 \%$ at 12 months post-immunization in a subsequent post hoc analysis. This trial was designed to prime with ALVAC-HIV, a canarypox vector, followed by a boost with AIDSVAX B/E, a bivalent subtype C gp120 protein, adjuvanted with aluminum hydroxide gel. Subsequent research has been done to identify the mechanism of protection conferred by this vaccine regimen. Immune correlates analyses revealed that those who were protected 
had enhanced complement deposition, high levels of $\operatorname{IgG} 1$ and $\operatorname{IgG} 3$ that targeted the variable loop regions 1 and 2 of gp120, lower IgA levels, and overall more polyfunctional immune response [19]. Unexpectedly, the concentration of neutralizing antibodies did not correlate with protection [47]. Other heterologous prime-boost regimens have revealed that a constellation of responses from the innate, adaptive, and cellular arms can be elicited. For instance, DNA platforms and viral vectors tend to elicit stronger $\mathrm{T}$ cell responses, whereas protein subunit antigens often provide a stronger humoral response. These prime-boost strategies appeared to add an important dimension to the multifaceted approach of potentiating a more effective response that was being achieved with modifications to antigens, adjuvants, or delivery platforms alone.

Despite early signs of modest efficacy in the RV144 trial, immunogenicity appeared to wane over time. As such, follow-on studies assessed the impact of late boosts with either ALVAC-HIV or AIDSVAX B/E®. ALVAC-HIV alone, however, did not induce significant HIV antibody responses, whereas participants who received ALVAC-HIV and AIDSVAX B/E $®$ or AIDSVAX B/E $®$ alone had antibody levels significantly higher than the peak levels seen in RV144 [48-51]. The latter boosting regimens, even when occurring 6-8 years later, promoted the expansion of lineages of broadly neutralizing antibodies through HCDR3 regions and greater somatic hypermutation.

Among the vaccines that have achieved emergency use authorization in the USA, to date, mRNA-1273 and BNT162b2 use homologous prime-boost regimens while Ad26.COV2.S is a single vaccine dose as the primary regimen. A subsequent set of "mix and match" studies that boosted individuals with a vaccine different from the original prime have shown a significant potentiation of both the cellular and humoral immune responses as compared to a homologous boost [52]. Longer-term evaluation will be needed to understand the relative durability and breadth of responses to differing vaccines. Lessons from the field of HIV vaccine development have demonstrated that heterologous prime-boost regimens generate a functional cell-mediated and humoral response. HIV research has demonstrated that combining viral vector or DNA vaccines with protein boosts show the most robust immune profiles.

\section{Immunologic Endpoints}

Assessment of vaccine potency, in the course of the development pipeline, is reliant upon standardized assays to assess immunologic responses. The earliest virus neutralization assays that used peripheral blood mononuclear cells (PBMC) and uncloned viruses were often cumbersome, expensive, and difficult to harmonize across laboratories
[53]. To address these limitations, assays with pseudotyped viruses were developed to evaluate antibody-mediated neutralization [54]. These same platforms were then repurposed for multiple viral pathogens, most recently for SARS-CoV-2. [55-57]. The use of pseudovirus assays has provided the additional benefit for viruses that require higher level biosafety level (BSL) containment, as these pseudoviruses only require BSL-2 conditions, which are more easily maintained in resource-limited settings [57].

Identification and harmonization of the appropriate immunologic endpoint assays are key to down-selecting and vetting promising vaccine candidates throughout the development pipeline. Advanced-phase clinical trials, however, are often necessary to determine protective efficacy of these vaccines. Ideally, protection or recovery from each infectious disease would correlate with a specific immunologic parameter that would inform the viability of vaccine candidates early in the evaluation process. These have been identified for multiple licensed vaccines in the past, serving as guidelines for the licensing of new vaccines. HIV research has demonstrated, however, that the elucidation of correlates of immunity can be difficult, particularly in the context of an infectious disease that is rarely cleared or cured. As stated earlier, some correlates of protection were identified in the RV144 for trial but, otherwise, markers of protective immunity have eluded HIV researchers [58]. However, the HIV field has paved a path by which correlates of immunity can be interrogated for other pathogens. A recent study, led in part by HIV researchers, demonstrated that threshold concentrations of neutralizing antibodies against SARS-CoV-2 correlated closely with protective efficacy for major COVID19 vaccines $[59,60]$. Identifying correlates of immunity will be important for next-generation COVID-19 vaccines, as these endpoints can reduce the need for large-scale efficacy trials and facilitate the rapid approval, licensure, and bridging of SARS-CoV-2 vaccines, particularly with continued emergence of SARS-CoV-2 variants.

\section{Variants}

The greatest obstacle to the development of an effective HIV vaccine has been the virus's high mutation rate and resultant immune evasion. SARS-CoV-2, in contrast, has a much lower mutation rate, thus restricting its genotypic and consequent phenotypic diversity [61]. In spite of its more limited variability, SARS-CoV-2 variants have been emerging, with significant impact on vaccine efficacy and other public health measures enacted to control the pandemic. The emergence of the Alpha, Beta, Delta, and Omicron variants has raised concerns about the effectiveness of vaccines based on the wild-type strain. Authorized or approved vaccines have had varying success against these variants. Despite the 
continued, though diminished effectiveness of these vaccines in preventing severe disease or death, continued development of second-generation vaccines is ongoing to prepare for the real threat of more variants, as evidenced by the recent emergence of the B.1.1.529/Omicron [62] strain. The emergence of this most recent and other viral variants of concern (i.e., Beta) highlights another link between the two topical viruses of this review, as SARS-CoV-2 infection may be prolonged in individuals living with HIV, who inherently have compromised immunity, thus providing favorable conditions for the evolution of more transmissible and antibodyresistant virus variants.

One approach that may offer an advantage in getting ahead of the emergence of new strains and species of coronaviruses is to develop multivalent vaccines that present an array of antigens in a single vaccine formulation so as to elicit a broadly protective response. Several groups are advancing nanoparticle platforms as the basis for this strategy. One in particular, developed by our group, is the SpFN vaccine adjuvanted with ALFQ, which is currently being evaluated in a clinical trial. The continued emergence of new strains and species of coronaviruses necessitates the advancement of these next-generation vaccine strategies that build on the innovation of HIV vaccine science. One of the key lessons from the HIV field is to take a holistic approach toward the generation and testing of new products, one that integrates the disciplines within the scientific community but also engages the participation of society at large.

\section{Community Engagement}

Success in containing the SARS-CoV-2 pandemic will rely, at least in part, on the availability, access, and uptake of vaccines. Buy-in on this and other public health measures across communities worldwide will be critical toward this end. Gains in grassroots advocacy and community empowerment made over the course of the HIV/AIDS pandemic provides poignant lessons and guidance for the current pandemic on how best to reach and recruit diverse groups, but particularly marginalized populations. Mirroring the HIV pandemic, COVID-19 has exacerbated health disparities in minority and underserved populations. In the USA, African American and Latinx communities have suffered a disproportionately higher rate of infections and adverse outcomes from COVID-19 [63-65]. Unsurprisingly, vaccine hesitancy, augmented by a long history of mistrust in public health institutions that has been amplified by misinformation, continues to be a barrier to vaccine uptake [66]. On a global scale, both the HIV/AIDS and COVID-19 pandemics

Table 1 Key advancements made in HIV vaccine research applied to COVID-19 vaccines

\begin{tabular}{|c|c|c|}
\hline Key advancement & Milestone in HIV vaccinology & Outcome for COVID19 vaccines \\
\hline \multirow[t]{3}{*}{ Vaccine platforms } & Nucleic acid platforms & $\begin{array}{l}\text { First vaccine candidates to receive EUA authorization } \\
\text { worldwide in }<1 \text { year }\end{array}$ \\
\hline & - Adenovirus vectors & $\begin{array}{l}\text { ChadOx-1 nCOV-19 } \\
\text { Ad26.COV2.S }\end{array}$ \\
\hline & - mRNA & $\begin{array}{l}\text { BNT162b2 } \\
\text { mRNA-1273 }\end{array}$ \\
\hline Structure-based antigen design & $\begin{array}{l}\text { Structure-based design of envelope ectodomain in tri- } \\
\text { meric prefusion conformation (BG505 SOSIP.664) }\end{array}$ & $\begin{array}{l}\text { Resolution of SARS-CoV-2 prefusion Spike glycopro- } \\
\text { tein within several weeks }\end{array}$ \\
\hline Adjuvants & $\begin{array}{l}\text { Failure of aluminum hydroxide prompts search for } \\
\text { new adjuvant formulations }\end{array}$ & $\begin{array}{l}\text { New aluminum formulations used in BBIP-CorV and } \\
\text { CoronaVac vaccines } \\
\text { Liposomal adjuvants used in combination with protein } \\
\text { subunit vaccines }\end{array}$ \\
\hline Dosing strategies & $\begin{array}{l}\text { Prime-boost regimens regain interest following results } \\
\text { of the RV144 trail }\end{array}$ & $\begin{array}{l}\text { Homologous, } 2 \text { dose, prime-boost for BNT162b2 and } \\
\text { mRNA-1273 receives EUA } \\
\text { Heterologous boosters are approved in the USA } \\
6 \text { months after completion of any vaccine regimen } \\
\text { against COVIS-19 }\end{array}$ \\
\hline Immunologic endpoints & $\begin{array}{l}\text { Standardized pseudovirus neutralization assays } \\
\text { Identification of IgG against V1V2 as correlate for } \\
\text { protection }\end{array}$ & $\begin{array}{l}\text { Repurposed to evaluate antibody neutralization of } \\
\text { SARS-CoV-2 } \\
\text { Correlates of immunity being identified }\end{array}$ \\
\hline Virus variants & $\begin{array}{l}\text { Expertise for tracking and evaluating mutation and } \\
\text { novel virus subtypes }\end{array}$ & $\begin{array}{l}\text { Surveillance network and scientific repurposed to mon- } \\
\text { itor and identify novel variants of interest/concern }\end{array}$ \\
\hline \multirow[t]{3}{*}{ Community engagement } & AVAC Good participatory Practice (GPP) & $\begin{array}{l}\text { GPP was used for community engagement in under- } \\
\text { served and hesitant populations }\end{array}$ \\
\hline & HVTN & CoVPN \\
\hline & PEPFAR & PEPVAR \\
\hline
\end{tabular}


have been complicated by international politics and the concern for equitable distribution of medical countermeasures [67]. Public health leaders recently have sought to apply the path of international health diplomacy navigated during the height of AIDS pandemic by referencing and adapting the AIDS Vaccine Advocacy Coalition (AVAC) Good Participatory Practice (GPP) guidelines to COVID-19 [68]. Lessons from AVAC's guidance documents highlight how an emerging virus could expose and exacerbate inequities in health outcomes [69]. Building on these lessons, researchers, public health practitioners, and community advocates quickly established the Community Engagement Alliance (CEAL) team and the COVID-19 Prevention Network (CoVPN) Community Engagement Working Group [70, 71]. Additionally, the G7 nations committed support early on for global vaccine procurement through the COVID-19 Vaccines Global Access (COVAX) initiative as means to ensure adequate supply vaccines to low- and middle-income countries (LMIC) [67, 72]. The initiative has fallen short of its goal so far and has not facilitated the widespread access to life-saving vaccines and treatments in the same way that the most successful HIV campaigns have provided, including the President's Emergency Plan for AIDS Relief (PEPFAR) and the Global Fund to Fight AIDS, Tuberculosis, and Malaria. A similar President's Emergency Plan for Vaccine Access and Relief or "PEPVAR," as coined in the media, would promote participation of well-resourced countries in favor of international partnerships for development and distribution of medical countermeasures [73].

The hard lessons from HIV were initially slow to realize and took significant effort to garner political and public support. With time, however, community engagement and advocacy proved to be essential in identifying and addressing systemic inequities that were hindering progress in the control of the HIV/AIDS pandemic: the same is being learned and applied in the current pandemic.

\section{Conclusion}

The COVID-19 pandemic has galvanized a vast and deep network of scientific expertise that owes much of its foundation to the advancements made in the fight against HIV. Prescient investments and prioritization of public health programs throughout the HIV pandemic have created a global enterprise that has been leveraged for the current campaign to control the COVID-19 pandemic. Despite the advantages gained from decades of prior groundwork, the public health response to SARS-CoV-2 has revealed gaps in preparedness. The key lessons learned from the current pandemic (Table 1), just as they did for HIV, will inform policies on how to better prepare for future public health threats.

\section{Declarations}

Conflict of Interest K. Modjarrad is a principal inventor of the SARS$\mathrm{CoV}-2$ Spike ferritin nanoparticle $(\mathrm{SpFN})$ vaccine.

Human and Animal Rights This article does not contain any studies with human or animal subjects performed by any of the authors.

Disclaimer Department of Defense.

The views expressed are those of the authors and should not be construed to represent the positions of the U.S. Army, the Department of Defense, or the Henry Jackson Foundation.

\section{References}

1. Wolff JA, et al. Direct gene transfer into mouse muscle in vivo. Science. 1990;247(4949 Pt 1):1465-8.

2. Sahin U, Karikó K, Türeci Ö. mRNA-based therapeuticsdeveloping a new class of drugs. Nat Rev Drug Discov. 2014;13(10):759-80.

3. Coughlan L, et al. Heterologous two-dose vaccination with Simian adenovirus and poxvirus vectors elicits long-lasting cellular immunity to influenza virus $\mathrm{A}$ in healthy adults. EBioMedicine. 2018;29:146-54.

4. Dicks MD, et al., A novel chimpanzee adenovirus vector with low human seroprevalence: improved systems for vector derivation and comparative immunogenicity. PLoS One, 2012. 7(7): e40385.

5. Barouch DH, et al. Evaluation of a mosaic HIV-1 vaccine in a multicentre, randomised, double-blind, placebo-controlled, phase 1/2a clinical trial (APPROACH) and in rhesus monkeys (NHP 13-19). Lancet. 2018;392(10143):232-43.

6. Sadoff J, et al., Interim Results of a Phase 1-2a Trial of Ad26. COV2.S Covid-19 Vaccine. New England Journal of Medicine, 2021.

7. Folegatti PM, et al. Safety and immunogenicity of the ChAdOx 1 nCoV-19 vaccine against SARS-CoV-2: a preliminary report of a phase $1 / 2$, single-blind, randomised controlled trial. The Lancet. 2020;396(10249):467-78.

8. Clinicaltrials.gov. Safety and immunogenicity study of DNA. HTI, MVA.HTI and ChAdOx1.HTI in HIV-1-positive Patients (AELIX-002). [cited 2021 7Jun]; Available from: https://clinicaltr ials.gov/ct2/show/NCT03204617.

9. Polack FP, et al. Safety and efficacy of the BNT162b2 mRNA Covid-19 vaccine. N Engl J Med. 2020;383(27):2603-15.

10. Baden LR, et al. Efficacy and safety of the mRNA-1273 SARSCoV-2 vaccine. N Engl J Med. 2020;384(5):403-16.

11. Gaschen B, et al. Diversity considerations in HIV-1 vaccine selection. Science. 2002;296(5577):2354-60.

12. Graham BS, Gilman MSA, McLellan JS. Structure-based vaccine antigen design. Annu Rev Med. 2019;70:91-104.

13. Rappuoli R. Reverse vaccinology. Curr Opin Microbiol. 2000;3(5):445-50.

14. Kwong PD, What are the most powerful immunogen design vaccine strategies? A structural biologist's perspective. Cold Spring Harb Perspect Biol, 2017. 9(11).

15. Ward AB, Wilson IA. Innovations in structure-based antigen design and immune monitoring for next generation vaccines. Curr Opin Immunol. 2020;65:50-6.

16. Liu $\mathrm{H}$, et al. The development of HIV vaccines targeting gp41 membrane-proximal external region (MPER): challenges and prospects. Protein Cell. 2018;9(7):596-615. 
17. Lyumkis D, et al. Cryo-EM structure of a fully glycosylated soluble cleaved HIV-1 envelope trimer. Science. 2013;342(6165):1484-90.

18. Julien JP, et al. Crystal structure of a soluble cleaved HIV-1 envelope trimer. Science. 2013;342(6165):1477-83.

19. Haynes BF, et al. Immune-correlates analysis of an hiv-1 vaccine efficacy trial. N Engl J Med. 2012;366(14):1275-86.

20. Sanders RW, Moore JP. Native-like Env trimers as a platform for HIV-1 vaccine design. Immunol Rev. 2017;275(1):161-82.

21. Sanders RW, et al., HIV-1 VACCINES. HIV-1 neutralizing antibodies induced by native-like envelope trimers. Science, 2015. 349(6244): p. aac4223.

22. Scheid JF, et al. Sequence and structural convergence of broad and potent HIV antibodies that mimic CD4 binding. Science. 2011;333(6049):1633-7.

23. McLellan JS, et al. Structure-based design of a fusion glycoprotein vaccine for respiratory syncytial virus. Science. 2013;342(6158):592-8.

24. McLellan JS, et al. Structure of RSV fusion glycoprotein trimer bound to a prefusion-specific neutralizing antibody. Science. 2013;340(6136):1113-7.

25. Stewart-Jones GB, et al., A cysteine zipper stabilizes a pre-fusion f glycoprotein vaccine for respiratory syncytial virus. PLoS One, 2015. 10(6): e0128779.

26. Joyce MG, et al. Iterative structure-based improvement of a fusion-glycoprotein vaccine against RSV. Nat Struct Mol Biol. 2016;23(9):811-20.

27. Krarup A, et al. A highly stable prefusion RSV F vaccine derived from structural analysis of the fusion mechanism. Nat Commun. 2015;6:8143.

28. Wrapp D, et al. Cryo-EM structure of the 2019-nCoV spike in the prefusion conformation. Science. 2020;367(6483):1260-3.

29. Pitisuttithum P, et al. Randomized, double-blind, placebo-controlled efficacy trial of a bivalent recombinant glycoprotein 120 HIV-1 vaccine among injection drug users in Bangkok Thailand. J Infect Dis. 2006;194(12):1661-71.

30. Rerks-Ngarm S, et al. Vaccination with ALVAC and AIDSVAX to prevent HIV-1 infection in Thailand. N Engl J Med. 2009;361(23):2209-20.

31. O'Connell RJ, et al. Safety and Immunogenicity of a Randomized Phase 1 Prime-Boost Trial With ALVAC-HIV (vCP205) and Oligomeric Glycoprotein 160 From HIV-1 Strains MN and LAI-2 Adjuvanted in Alum or Polyphosphazene. J Infect Dis. 2016;213(12):1946-54.

32. Weissburg RP, et al. Characterization of the MN gp120 HIV-1 vaccine: antigen binding to alum. Pharm Res. 1995;12(10):1439-46.

33. Flynn NM, et al. Placebo-controlled phase 3 trial of a recombinant glycoprotein 120 vaccine to prevent HIV-1 infection. J Infect Dis. 2005;191(5):654-65.

34. Pegu P, et al. Antibodies with high avidity to the gp120 envelope protein in protection from simian immunodeficiency virus SIV(mac251) acquisition in an immunization regimen that mimics the RV-144 Thai trial. J Virol. 2013;87(3):1708-19.

35. Tuero I, et al., Mucosal B cells are associated with delayed SIV acquisition in vaccinated female but not male rhesus macaques following SIVmac251 rectal challenge. PLoS Pathog, 2015. 11(8): e1005101.

36. Fouts TR, et al. Balance of cellular and humoral immunity determines the level of protection by HIV vaccines in rhesus macaque models of HIV infection. Proc Natl Acad Sci U S A. 2015;112(9):E992-9.

37. Iyer SS, et al. Codelivery of envelope protein in alum with MVA vaccine induces CXCR3-Biased CXCR5+ and CXCR5CD4 T Cell Responses in Rhesus Macaques. J Immunol. 2015;195(3):994-1005.
38. Barouch DH, et al. Protective efficacy of adenovirus/protein vaccines against SIV challenges in rhesus monkeys. Science. 2015;349(6245):320-4.

39. Apostólico Jde S, et al., HIV envelope trimer specific immune response is influenced by different adjuvant formulations and heterologous prime-boost. PLoS One, 2016. 11(1): e0145637.

40. Leroux-Roels I, et al. Strong and persistent CD4+ T-cell response in healthy adults immunized with a candidate HIV-1 vaccine containing gp120, Nef and Tat antigens formulated in three Adjuvant Systems. Vaccine. 2010;28(43):7016-24.

41. Alving CR, et al. Liposomal adjuvants for human vaccines. Expert Opin Drug Deliv. 2016;13(6):807-16.

42. Beck Z, Matyas GR, Alving CR. Detection of liposomal cholesterol and monophosphoryl lipid A by QS-21 saponin and Limulus polyphemus amebocyte lysate. Biochim Biophys Acta. 2015;1848(3):775-80.

43. Beck Z, et al. Differential immune responses to HIV-1 envelope protein induced by liposomal adjuvant formulations containing monophosphoryl lipid A with or without QS21. Vaccine. 2015;33(42):5578-87.

44. Wieczorek L, et al. Comparable antigenicity and immunogenicity of oligomeric forms of a novel, acute HIV-1 subtype C gp145 envelope for use in preclinical and clinical vaccine research. J Virol. 2015;89(15):7478-93.

45. Clinicaltrials.gov. HIV Vaccine in HIV-uninfected Adults. [cited 2021 7Jun]; Available from: https://clinicaltrials.gov/ct2/show/ NCT04658667.

46. Robb ML, et al. Risk behaviour and time as covariates for efficacy of the HIV vaccine regimen ALVAC-HIV (vCP1521) and AIDSVAX B/E: a post-hoc analysis of the Thai phase 3 efficacy trial RV 144. Lancet Infect Dis. 2012;12(7):531-7.

47. Zolla-Pazner $\mathrm{S}$, et al., Analysis of V2 antibody responses induced in vaccinees in the ALVAC/AIDSVAX HIV-1 vaccine efficacy trial. PLoS One, 2013. 8(1): p. e53629.

48. Rerks-Ngarm S, et al. Randomized, double-blind evaluation of late boost strategies for HIV-Uninfected vaccine recipients in the RV144 HIV vaccine efficacy trial. J Infect Dis. 2017;215(8):1255-63.

49. Easterhoff D, et al., Boosting of HIV envelope CD4 binding site antibodies with long variable heavy third complementarity determining region in the randomized double blind RV305 HIV-1 vaccine trial. PLoS Pathog, 2017. 13(2): p. e1006182.

50. Easterhoff D, et al. Boosting with AIDSVAX B/E enhances Env constant region 1 and 2 antibody-dependent cellular cytotoxicity breadth and potency. J Virol, 2020. 94(4).

51. Easterhoff D, et al. HIV vaccine delayed boosting increases Env variable region 2 -specific antibody effector functions. JCI Insight, 2020. 5(2).

52. Atmar RL, et al. Heterologous SARS-CoV-2 booster vaccinations - preliminary report. medRxiv, 2021.

53. Montefiori D, et al. Antibody-based HIV-1 vaccines: recent developments and future directions. PLoS Med, 2007. 4(12): e348.

54. Polonis VR, et al. Recent advances in the characterization of HIV-1 neutralization assays for standardized evaluation of the antibody response to infection and vaccination. Virology. 2008;375(2):315-20.

55. Joyce MG, et al. Efficacy of a broadly neutralizing SARS-CoV-2 ferritin nanoparticle vaccine in nonhuman primates. bioRxiv, 2021: p. 2021.03.24.436523.

56. Weissman D, et al. D614G spike mutation increases SARS CoV-2 susceptibility to neutralization. Cell Host Microbe. 2021;29(1):23-31.e4.

57. Sholukh AM, et al. Evaluation of SARS-CoV-2 neutralization assays for antibody monitoring in natural infection and vaccine trials. medRxiv, 2020. 
58. Alter G, Barouch D. Immune correlate-guided hiv vaccine design. Cell Host Microbe. 2018;24(1):25-33.

59. Gilbert PB, et al. Immune correlates analysis of the mRNA1273 COVID-19 vaccine efficacy clinical trial. Science, 2021: p. eab3435.

60. Khoury DS, et al. Neutralizing antibody levels are highly predictive of immune protection from symptomatic SARS-CoV-2 infection. Nat Med. 2021;27(7):1205-11.

61. Dearlove B, et al. A SARS-CoV-2 vaccine candidate would likely match all currently circulating variants. Proc Natl Acad Sci U S A. 2020;117(38):23652-62.

62. Wu K, et al. Variant SARS-CoV-2 mRNA vaccines confer broad neutralization as primary or booster series in mice. bioRxiv, 2021: p. 2021.04.13.439482.

63. CDC. Risk for COVID-19 Infection, hospitalization and death by race/ethnicity. 202123 Apr 2021 [cited 202110 May 2021]; Available from: https://www.cdc.gov/coronavirus/2019-ncov/ covid-data/investigations-discovery/hospitalization-death-byrace-ethnicity.html?CDC_AA_refVal=https $\% 3 \mathrm{~A} \% 2 \mathrm{~F} \% 2 \mathrm{Fwww}$. cdc.gov\%2Fcoronavirus\%2F2019-ncov\%2Fcommunity\%2Fhea lth-equity $\% 2$ Fracial-ethnic-disparities $\% 2$ Finfographic-cases-hospi talization-death.html.

64. Williamson EJ, et al. Factors associated with COVID-19-related death using OpenSAFELY. Nature. 2020;584(7821):430-6.

65. Webb Hooper M, Nápoles AM, Pérez-Stable EJ, COVID-19 and racial/ethnic disparities. JAMA, 2020. 323(24): p. 2466-2467.
66. Weintraub RL, et al. COVID-19 vaccine to vaccination: why leaders must invest in delivery strategies now. Health Aff (Millwood). 2021;40(1):33-41.

67. Katz IT, et al. From vaccine nationalism to vaccine equity - finding a path forward. N Engl J Med. 2021;384(14):1281-3.

68. AVAC. Applying global health advocacy to the COVID-19 response. [cited 2021 7Jun]; Available from: https://www.avac. org/covid.

69. Killen J, Harrington M, Fauci AS. MSM, AIDS research activism, and HAART. Lancet. 2012;380(9839):314-6.

70. CoVPN. COVID-19 Prevention Network About Us. 202111 May 2021]; Available from: https://www.coronaviruspreventionnetw ork.org/about-covpn/.

71. CEAL. Community Engagement Alliance at a Glance. 11 May 2021]; Available from: https://covid19community.nih.gov/about.

72. Yamey G, et al. Ensuring global access to COVID-19 vaccines. Lancet. 2020;395(10234):1405-6.

73. NYTimes. The World is desperate for more COVID vaccines. Available from: https://www.nytimes.com/2021/01/12/opinion/ world-covid-vaccines.html.

Publisher's Note Springer Nature remains neutral with regard to jurisdictional claims in published maps and institutional affiliations. 